\title{
VLT spectroscopy of galaxies lensed by the AC 114 cluster:
}

\section{Implications for the mass model and the study of low-luminosity galaxies at high-redshift ${ }^{\star}$}

\author{
L. E. Campusano ${ }^{1}$, R. Pelló ${ }^{2}$, J.-P. Kneib ${ }^{2}$, J.-F. Le Borgne ${ }^{2}$, B. Fort ${ }^{3}$, R. Ellis ${ }^{4}$, \\ Y. Mellier ${ }^{3,5}$, and I. Smail ${ }^{6}$
}

1 Observatorio Astronómico Cerro Calán, Departamento de Astronomía, U. de Chile, Casilla 36-D, Santiago, Chile

2 Observatoire Midi-Pyrénées, UMR 5572, 14 avenue E. Belin, 31400 Toulouse, France

3 Institut d'Astrophysique de Paris, 98 bis boulevard Arago, 75014 Paris, France

4 Astronomy 105-24, Caltech, Pasadena, CA 91125, USA

5 Observatoire de Paris, DEMIRM, 61 avenue de l'Observatoire, 75014 Paris, France

${ }^{6}$ Department of Physics, University of Durham, South Road, Durham DH1 3LE, UK

Received 24 April 2001 / Accepted 14 August 2001

\begin{abstract}
We present the first results of a spectroscopic survey of faint lensed galaxies in the core of the galaxy cluster AC114 ( $z=0.312)$ obtained from observations with the FORS1 spectrograph mounted on the VLT-Antu (Unit Telescope 1). The galaxies were chosen according to both lensing and photometric redshift criteria in areas close to the high- $z$ critical lines predicted by the gravitational lens model of Natarajan et al. (NKSE, 1998) for this cluster. All the target galaxies are found to correspond to background galaxies with redshift values in the [0.7, 3.5] interval. Our spectroscopic observations confirm the predicted lensing redshifts for 3 of the multiply-imaged galaxies, and together with predictions of the NKSE model led to the discovery of a new 5-image configuration at redshift $z=3.347$. A revised NKS model, compatible with the redshift of this new multiple-image system, was generated and employed to calculate the gravitational amplifications of all the observed galaxies. The galaxies corresponding to the multiple-image systems are found to be intrinsically fainter, between 0.5 and 1.5 magnitudes, than the limiting magnitudes of existing blank field studies. When all the observed background galaxies are considered, the resulting intrinsic absolute magnitudes range from $M_{B} \sim-22$ to -19 , with a median value of -20.5. Therefore, a large gain in sensitivity towards low luminosity high- $z$ objects can actually be obtained, in agreement with theoretical expectations. This method can be used advantageously to probe the high redshift Universe and, in particular, its application to an ensemble of massive cluster cores could constraint the faint end of luminosity function of high redshift galaxies.
\end{abstract}

Key words. cosmology: observations - galaxies: clusters: individual (AC 114) - galaxies: fundamental parameters gravitational lensing - galaxies: redshifts - general

\section{Introduction}

The investigation of the properties of distant galaxies can be conducted by observing them either in blank fields or through the cores of massive clusters of galaxies. Cluster lenses are natural telescopes gravitationally magnifying the background galaxies, thus they allow the detection of intrinsically fainter and more distant galaxies than otherwise possible. Of particular interest is the study of spectroscopically confirmed high- $z$ galaxies in cluster cores where

Send offprint requests to: L. Campusano,

e-mail: lcampusa@das.uchile.cl

* Based on observations collected with the ESO Very Large Telescope Antu (UT1) and NTT $3.5 \mathrm{~m}$ Telescope, and the Hubble Space Telescope. the amplification is the highest, attaining typically values from 1 to 3 magnitudes. A sample of these galaxies would extend to fainter magnitudes than the ones currently available from surveys of the field (Steidel et al. 1996a, 1996b, 1998, 1999), and also complement the latter, because it would be less biased towards intrinsically brighter galaxies. The evolution of the luminosity function (LF) with redshift is mainly characterized by a brightening of the luminosity with look-back time, with significant effects at around $M_{B} \sim-20$ involving the faintest and bluest galaxies in field samples (Lilly et al. 1995; Ellis et al. 1996; Lilly et al. 1998). The luminosity density of star-forming galaxies, as traced by [OII] emission, also increases with redshift up $z \sim 1$, and an important fraction of such star-forming activity is located in low-luminosity galaxies 
Table 1. Characteristics of the images and detection levels. $3 \sigma$ magnitudes correspond to objects with 4 connected pixels, each $3 \sigma$ above the sky level. Observations were carried out at the HST (1), ESO NTT (2), the CTIO $4 \mathrm{~m}$ telescope (3) and the the AAT (4).

\begin{tabular}{lllrrrrr}
\hline & $\begin{array}{l}t_{\exp } \\
(\mathrm{ksec})\end{array}$ & $\begin{array}{l}\sigma \\
\left({ }^{\prime \prime}\right)\end{array}$ & $\begin{array}{r}\text { pix } \\
\left({ }^{\prime \prime}\right)\end{array}$ & $\begin{array}{r}\lambda_{\text {eff }} \\
(\mathrm{nm})\end{array}$ & $\begin{array}{r}\Delta \lambda \\
(\mathrm{nm})\end{array}$ & $\begin{array}{r}m \\
3 \sigma\end{array}$ & Ref. \\
\hline$U$ & 20.0 & 1.3 & 0.36 & 365 & 40 & 27.1 & $\mathrm{a}, 3$ \\
$B$ & 9.0 & 1.2 & 0.39 & 443 & 69 & 27.7 & 4 \\
$V$ & 21.6 & 1.1 & 0.47 & 547 & 53 & 26.7 & $\mathrm{~b}, 2$ \\
$V_{555}$ & 20.7 & 0.3 & 0.10 & 545 & 105 & 25.8 & $\mathrm{~b}, 1$ \\
$R$ & 16.8 & 0.13 & 0.10 & 694 & 122 & 25.7 & $\mathrm{c}, 1$ \\
$I_{814}$ & 20.7 & 0.3 & 0.10 & 801 & 134 & 24.9 & $\mathrm{~b}, 1$ \\
$J$ & 7.2 & 0.9 & 0.29 & 1253 & 169 & 21.5 & 2 \\
$K^{\prime}$ & 10.8 & 0.8 & 0.29 & 2164 & 164 & 20.0 & 2 \\
\hline
\end{tabular}

a Barger et al. (1996), ${ }^{\mathrm{b}}$ Smail et al. (1991), ${ }^{\mathrm{c}}$ Natarajan et al. (1998).

(Ellis et al. 1996), with a significant contribution of mergers (Le Fèvre et al. 2000). In order to address the evolution of the different populations of galaxies, a large sample is needed at each redshift, spanning a wide range of luminosity, ideally down to sub-L* levels. Given the difficulty of attaining in this goal through current apparent-magnitude limited field samples, it is important to take advantage of the gravitational amplification so as to i) detect high redshift galaxies more easily, and ii) to probe the faint end of the luminosity function of high redshift galaxies.

Natural telescopes have been successfully used in studying distant galaxies at almost all wavebands from the UV (Bézecourt et al 1999), Optical (e.g. Ebbels et al. 1998; Pelló et al. 1999a, 1999b), Mid-Infrared (Altieri et al. 1999; Metcalfe et al. 1999) to Submm (Smail et al. 1997; Ivison et al. 2000). A difficulty of the optical/near-infrared, much less critical in other wavebands, is the contamination by cluster galaxies which are not always easily separated from the background population. Furthermore, because of the shallow slope of the galaxy number counts at faint magnitude, the magnification effect dilutes the density of background galaxies in the visible/near-infrared bands, hence for a given magnitude limit, there will be effectively fewer galaxies seen through a cluster lens than in blank fields (Broadhurst 1995; Fort et al. 1997; Mayen \& Soucail 2000). For studies of the luminosity function of high redshift galaxies, a large number (typically $\sim 100$ ) of these galaxies are needed. Such a number can be collected through observations, like the ones reported here, of a sample of about 10 massive cluster-lenses. Our selection criteria for the distant galaxy candidates are based on a combination of lensing (Kneib et al. 1994, 1996; Ebbels et al. 1998) and photometric redshift criteria (e.g. Mobasher et al. 1996; Lanzetta et al. 1996; Gwyn \& Hartwick 1996; Sawicki et al. 1997; Giallongo et al. 1998; Fernández-Soto et al. 1999, Arnouts et al. 1999;
Furusawa et al. 2000; Bolzonella et al. 2000). Although the lensing criteria in the cluster core is sufficient (once the mass distribution of the cluster is well determined) for the selection of distant galaxies (Ebbels et al. 1998), photometric redshifts are very effective to select them in the outer part of the cluster and are also essential in identifying distant galaxies not resolved by $H S T$ and located in the cluster core. Furthermore, the photometric properties of the faint galaxies helps to optimize the instrument choice for the spectroscopic follow-up (visible vs. near-IR bands).

In this paper, we focus on a particularly interesting cluster-lens, AC 114 (also named ACO S1077, Abell et al. 1989), a $z=0.312$ rich cluster showing a large number of multiple-images at high- $z$ (Smail et al. 1995; Natarajan et al. 1998, hereafter NKSE). The redshift of the gravitational pair S1/S2, $z=1.86$, was obtained by Smail et al. (1995) with AAT, and later confirmed with NTT with a total exposure time of $\sim 10$ hours. Based on this redshift, the detailed mass-model derived by NKSE predicts the redshifts of 4 multiple images $(A, B, C$ and $D)$, ranging from $z \sim 1$ to 2.5. Here, we present the results obtained from a spectroscopic survey of lensed galaxies in AC 114 conducted with VLT/FORS1 at Paranal (program 64.O0439A). Sections 2 and 3 present a summary of the observations, selection criteria and data reduction. A detailed discussion of the spectroscopic results is given in Sect. 4. In Sect. 5, we briefly discuss the improved lens model for AC 114, which now includes the spectroscopic redshift of 2 multiple images as constraints. The properties of these amplified galaxies are described in Sect. 6. Discussion and conclusions are given in Sects. 7 and 8. Throughout this paper, we adopt $H_{0}=50 \mathrm{~km} \mathrm{~s}^{-1} \mathrm{Mpc}^{-1}, \Omega_{\mathrm{m}}=0.3$ and $\Omega_{\Lambda}=0.7$.

\section{Imaging data and target selection}

All the publically available images of the cluster core of AC 114, to October 1999, were employed for the selection of background galaxies. These data include deep $H S T$ images in 3 filter bands: $\mathrm{F} 555 \mathrm{~W} / W F P C\left(V_{555}\right)$, F702W/WFPC2 $(R)$, and F814W/WFPC $\left(I_{814}\right)$, as well as $U$ (CTIO) and $V$ (ESO-NTT) images. In addition, we used deep imaging from a related program with ESO-NTT and the SOFI camera, including both $J$ and $K$ bands. Unfortunatedly, a photometric run with SUSI, aimed at obtaining $B$ photometry, failed because of weather. After our VLT spectroscopic run, we got an existing deep $B$ image (courtesy of W. J. Couch), which is taken into consideration for the discussion about the photometric and lensing redshifts of the spectroscopically observed galaxies. The basic characteristics of these images are listed in Table 1, including magnitude limits, and references.

In the very central part of the cluster, the high-redshift galaxy candidates were mainly selected using lensing criteria (e.g. NKSE). Photometric redshifts were primarily used for the selection of background galaxies lying on the outer part of the cluster core, where lensing criteria are much less efficient, as well as in the core for cases when 


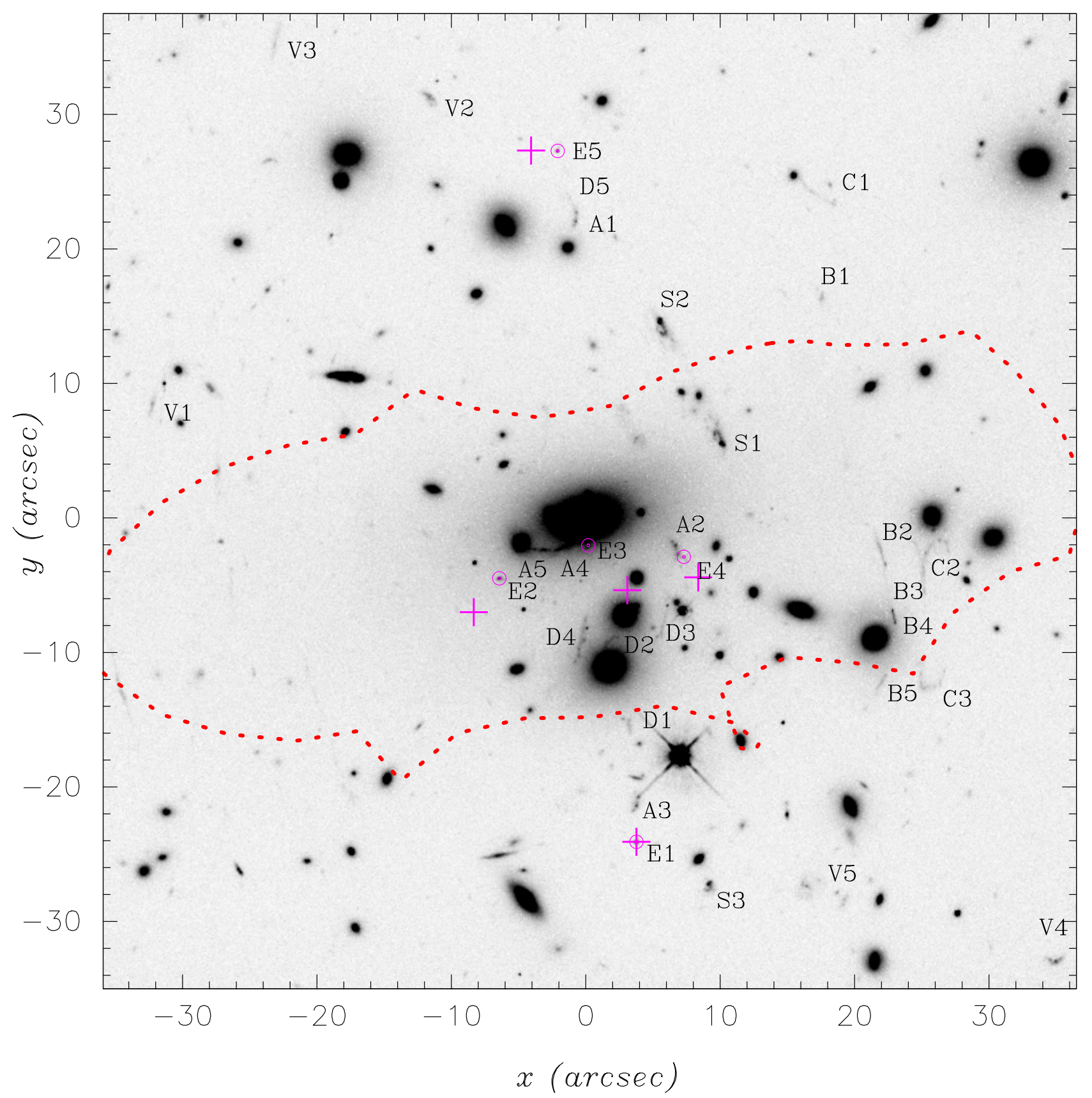

Fig. 1. HST/WFPC2 image (F702W) of AC 114 cluster core with the identified high redshift galaxies and multiple images. The critical lines (dotted lines) are shown for $z=3.35$. Circles show the position of the observed E system, and crosses correspond to the positions predicted by the NKSE model for the counter images of E1.

lensing failed to give any prediction - specially when the image of the galaxy was barely resolved. The photometric catalogue was constructed, after matching the seeing values on the different images, using the SExtractor package (Bertin \& Arnouts 1996). Magnitudes in this paper refer to the Vega system. Photometric redshifts (hereafter $\left.z_{\text {phot }}\right)$ were computed through a standard minimization procedure, using the hyperz code (Bolzonella et al. 2000). This procedure uses a template library of spectra mainly derived from the new Bruzual \& Charlot evolutionary code (GISSEL98, Bruzual \& Charlot 1993). Photometric redshifts were derived from magnitudes computed within a $4^{\prime \prime}$ diameter aperture. In the case of highly distorted images, located close to bright cluster members, such as A, $\mathrm{B}$ and $\mathrm{C}$, photometry was obtained through special apertures adapted to the shape of each object so that the same physical region was considered for the magnitude calculations in all the available images. Because of the lack of a $B$-band image, the error bars of the photometric redshifts employed in the target selection were found to be typically $\sigma_{z} \sim \pm 0.2$ to 0.3 , with degenerate solutions in some cases. Given that the CCD images cover fields of different size, the photometric selection could be done effectively only on the central $H S T$ field covering $\sim 80^{\prime \prime} \times 80^{\prime \prime}$. Twelve galaxy candidates, with $z \geq 1.0$ and $R<24$ were identified in this region. 
Table 2. Position and photometry for the 23 observed background galaxies. The horizontal line separates the core sample, for which the strong selection criteria apply. When available, the identifications are given according to Natarajan et al. 1998. "V" objects have numbers increasing with their distance from the cluster center. When a space is left blank, the object is "out of the field" in this filter, whereas non-detected objects are given by "-". V-band photometry corresponds to ground-based data. These are aperture magnitudes computed within a $4^{\prime \prime}$-diameter aperture, after correction for seeing differences between the images.

\begin{tabular}{cccccccccc}
\hline Id. & RA (J2000.0) & DEC. (J2000.0) & $U$ & $B$ & $V$ & $R$ & $I_{814}$ & $J$ & $K^{\prime}$ \\
\hline A1 & $22: 58: 49.58$ & $-34: 47: 53.0$ & 23.72 & 23.37 & 22.35 & 23.07 & 23.32 & 22.07 & 21.43 \\
A2 & $22: 58: 47.77$ & $-34: 48: 04$. & & & & & & & \\
B2 & $22: 58: 46.80$ & $-34: 47: 54.8$ & 23.66 & 24.02 & 23.28 & 22.48 & & 22.54 & $22.25:$ \\
B3 & $22: 58: 46.53$ & $-34: 47: 57.1$ & & & & & & & \\
C3 & $22: 58: 46.11$ & $-34: 47: 59.2$ & 24.68 & 24.37 & 23.59 & 22.44 & 23.66 & - & - \\
E1 & $22: 58: 46.67$ & $-34: 48: 22.4$ & - & 25.06 & 24.32 & 23.77 & 23.59 & - & 22.68 \\
S2 & $22: 58: 48.78$ & $-34: 47: 54.0$ & 22.46 & 22.81 & 22.54 & 22.01 & 22.10 & 21.04 & 19.99 \\
V1 & $22: 58: 50.59$ & $-34: 48: 24.9$ & 24.27 & 24.77 & 24.69 & 23.94 & 23.23 & 21.59 & 19.80 \\
V2 & $22: 58: 50.72$ & $-34: 47: 54.3$ & 23.49 & 24.27 & 23.91 & 23.20 & 22.84 & 21.71 & 21.06 \\
V3 & $22: 58: 51.61$ & $-34: 47: 59.6$ & 24.70 & 25.31 & 24.94 & 23.79 & 22.78 & 21.04 & 19.37 \\
V4 & $22: 58: 44.39$ & $-34: 48: 06.7$ & 24.06 & 24.42 & 23.97 & 23.06 & 23.28 & 22.45 & 21.03 \\
V5 & $22: 58: 45.75$ & $-34: 48: 15.8$ & 23.80 & 24.50 & 23.84 & 22.97 & 22.68 & 21.10 & 19.25 \\
\hline V6 & $22: 58: 50.94$ & $-34: 47: 26.5$ & & 23.56 & 22.58 & 21.77 & 20.95 & 20.03 & 18.73 \\
V7 & $22: 58: 45.60$ & $-34: 49: 03.9$ & 22.88 & 22.97 & 22.16 & 20.66 & 20.15 & 19.17 & 17.68 \\
V8 & $22: 58: 54.12$ & $-34: 48: 28.2$ & & 23.71 & & & & 20.37 & 18.92 \\
V9 & $22: 58: 56.56$ & $-34: 46: 58.6$ & & 22.67 & & & & & 17.00 \\
V10 & $22: 58: 56.98$ & $-34: 48: 45.8$ & & 25.29 & & & & & 17.72 \\
V11 & $22: 58: 57.46$ & $-34: 47: 06.8$ & & 23.61 & & & & & 18.39 \\
V12 & $22: 58: 38.15$ & $-34: 48: 25.3$ & & 22.84 & & 20.58 & & 19.30 & 17.43 \\
V13 & $22: 58: 38.70$ & $-34: 50: 08.2$ & & 24.24 & & & & 20.67 & 18.69 \\
V14 & $22: 58: 38.71$ & $-34: 50: 08.5$ & & 24.04 & & & & 21.60 & 19.77 \\
V15 & $22: 58: 36.21$ & $-34: 49: 40.5$ & & 25.28 & & & & & 17.03 \\
V16 & $22: 58: 34.59$ & $-34: 49: 30.1$ & & 23.08 & & & & & 18.15 \\
V17 & $22: 58: 34.80$ & $-34: 50: 34.1$ & & 26.26 & & & & & 18.75 \\
V18 & $22: 58: 33.65$ & $-34: 49: 42.2$ & & 23.40 & & & & & \\
\hline & & & & & & & & & \\
\hline
\end{tabular}

Given that both lensing and photometric redshifts were available for most of the high- $z$ galaxy candidates lying in the cluster core, and that the spectroscopic setup allowed the observation of only a few of them, we proceeded mostly with the candidates with consistent redshift predictions. The designation of the observed objects is the same as that of NKSE, when available, otherwise they are labelled " $V$ " with numbers increasing with their distance from the cluster center. The galaxies in the core that were actually observed, are identified in Fig. 1. In Table 2, positions and $U B V R I J K$ photometry are given for the all the observed background galaxies.

\section{Spectroscopic observations and data reduction}

The spectra of the high-redshift galaxy candidates were obtained on the night of October 5, 1999, with the ESO VLT Antu (UT1) telescope and its FORS1 spectrograph working in the multi-object mode. A slit width of $1^{\prime \prime}$ and the grism G300V were used, resulting in a wavelength coverage of $\sim 4000-8600 \AA$, and a resolution of $R=500$. Three masks, or slit configurations, were constructed and used with exposure times of $2 \mathrm{~h} 15 \mathrm{~m}, 1 \mathrm{~h} 30 \mathrm{~m}$ and $1 \mathrm{~h} 17 \mathrm{~m}$, respectively. Each of them spans a $\sim 7^{\prime}$ field, with 19 to 20 slitlets having a fixed $22^{\prime \prime}$ length. The fact that the slit length was comparable to the angular size of the cluster core, restricted to $\sim 5-6$ the number of strongly magnified galaxy candidates that could be observed per mask. Multiple images according to the NKSE model were given the first priority, and the secondary targets to fill the remaining slitlets inside the $1.2^{\prime} \times 1.2^{\prime}$ cluster core were chosen from the photometric high- $z$ list of candidates. In order to improve the efficiency, we kept some of the faintest candidates from the cluster core in several slit configurations while changing the brighter targets lying in the outer parts of the cluster. Once the priority targets had determined the position of the center of the mask and its orientation, the slits outside the core could be used only to observe galaxy cluster members. The seeing values during the observations were in the range $\sim 0.6-0.8^{\prime \prime}$, except during the last $1500 \mathrm{~s}$ of the exposure with the third mask $\left(1.3^{\prime \prime}\right)$. Spectra of the standard star Feige 110 were obtained during twilight for calibration. The data reduction was done using standard IRAF packages. 


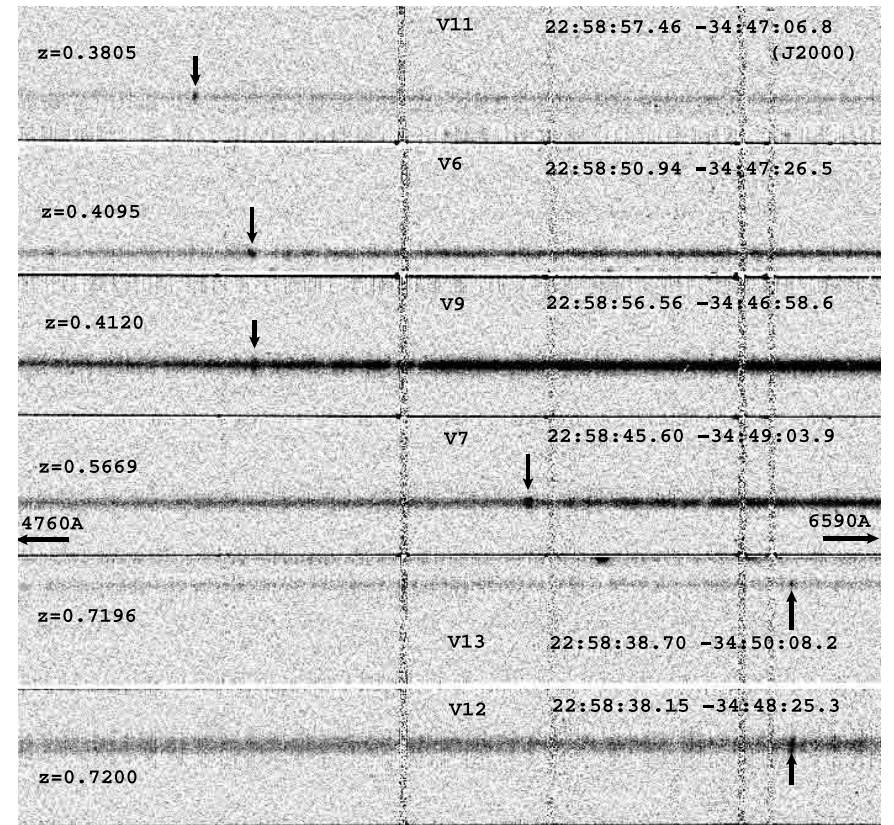

Fig. 2. Two dimensional spectra of background galaxies showing emission lines in the low redshift domain: $0.33 \leq z \leq 0.80$. Galaxies are identified as in Table 2. The arrows indicate the position of $[\mathrm{OII}] 3727 \AA$ line. The wavelength interval $4760 \AA-$ $6590 \AA$ is shown.

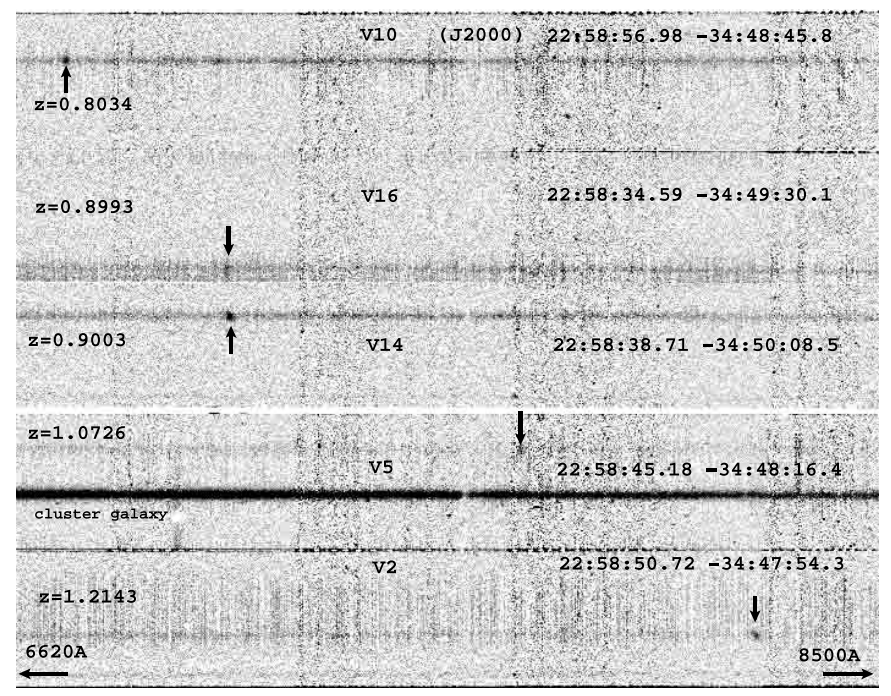

Fig. 3. Two dimensional spectra of background galaxies showing emission lines in the high redshift domain: $0.80 \leq z \leq 1.21$. Galaxies are identified as in Table 2. The arrows indicate the position of [OII] line at $3727 \AA$. The wavelength interval $6620 \AA-8500 \AA$ is shown.

\section{Spectroscopic results}

Sixty-two galaxy spectra were extracted from the integrations performed through the 3 mask configurations selected for use with FORS1. The inspection of the wavelength and flux calibrated spectra revealed that twentyseven were galaxies belonging to AC 114, twenty-three galaxies lay in the background of the cluster, and ten were foreground galaxies (two spectra remained unidentified). In this paper, we concentrate on the spectroscopically identified background galaxies and, in particular, on those located in the central $1.2^{\prime} \times 1.2^{\prime}$ region of $\mathrm{AC} 114$. The results on the cluster galaxies will be presented in a forthcoming paper.

The 2-dimensional spectra of the background galaxies with $0.33 \leq z \leq 0.80$ are shown in Fig. 2, where the arrows indicate the position of the [OII] line at $3727 \AA$. Similarly, Fig. 3 displays the 2-dimensional spectra for the $0.80 \leq z \leq 1.21$ interval. Figure 4 displays calibrated onedimensional spectra of the observed background galaxies with $z \gtrsim 1$, showing the identified emission and absorption features. The designation of the galaxies is the same as indicated in Table 2.

The redshifts measured for the background galaxies are listed in Table 3, together with the identified spectral features either in absorption or emission. For the objects with a fair signal-to-noise value, the best fit galaxy spectral type is given, from $\mathrm{E}$ to $\mathrm{Im}$. A discussion of the redshift determinations of the galaxies lying in the cluster core is given below, which is specially delicate for the spectra presenting only one strong spectral feature. Table 3 summarizes the redshift determinations and the main spectral features identified. Excepting one uncertain case (V1, see Sect. 6.5), all of the 10 high- $z$ candidates selected in the cluster core region were found to have spectroscopic redshifts between $\sim 1$ and 3.5, in good agreement with the redshift selection criteria. The strongly lensed galaxies in the cluster core are discussed individually in Sect. 6 .

For comparison, Table 5 lists the lensing (NKSE) redshifts for the multiple systems and the photometric redshifts for the the observed galaxies in the cluster core, all of them with predicted redshifts greater than $\sim 1$. The only exception is $\mathrm{V} 3$, for which the a posteriori $z_{\text {phot }}$ is lower than the initial estimate, $z \sim 0.8$, and in fair agreement with the spectroscopic value. Overall, our method to identify lensed galaxies with $z>1.0$ had a success rate $\gtrsim 80 \%$. Although the $z_{\text {phot }}$ used for target selection had larger uncertainties than those given in Table 5 (because of the lack of deep $B$ photometry), they were an effective complement to the $z_{\text {lens }}$ specially in the case of barely resolved objects which did not have a predicted $z_{\text {lens }}$, and turned out to be fully compatible with the spectroscopic redshift within the errors. Table 5 also lists the $z_{\text {phot }}$ for the rest of the observed background galaxies.

From Table 5 , it can be seen that the $z_{\text {lens }}$ predicted by the NKSE for A1/A2 is in remarkable agreement with the measured redshift for the system. Although the redshift determination of the systems B and C is more uncertain, they suggest that the $z_{\text {lens }}$ predictions of the NKSE underestimate the actual values.

The object designated E1 in Table 3, showing a strong emission line with $z=3.347$ when identified with Ly $\alpha$ (see Fig. 8), provided a surprising prediction of the NKSE lens model, which to a large extent was confirmed by the available data. E1, was selected as a high- $z$ galaxy based solely on its photometric properties; it did not have a $z_{\text {lens }}$ 

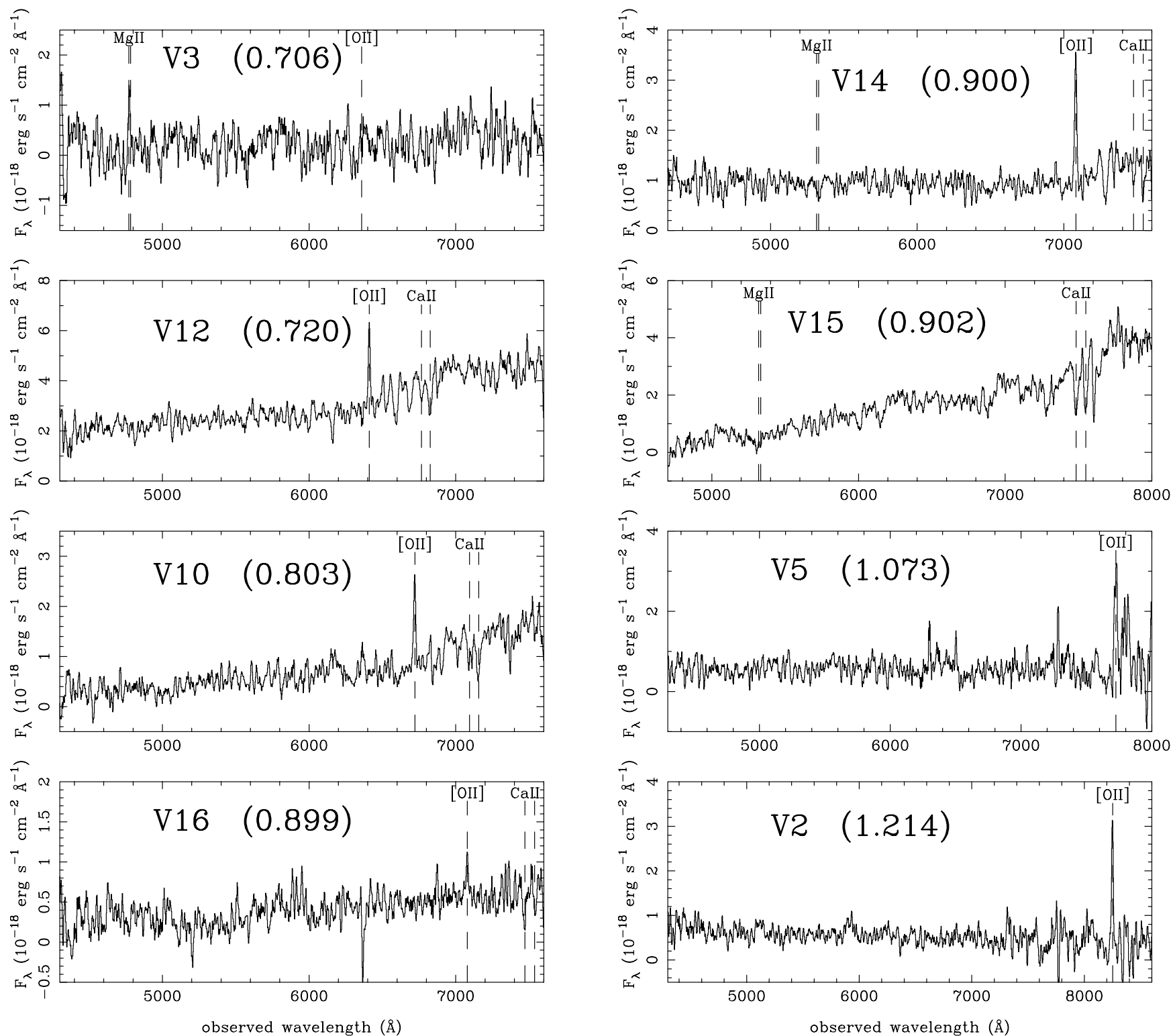

Fig. 4. Spectra of the galaxies in the background of AC 114 having the best signal-to-noise ratios. The label on the top of each spectrum gives the identification number of the galaxy, according to Table 2, and its measured redshift. Fluxes are given in arbitrary units, but provide the right order of magnitude in ergs s $\mathrm{cm}^{-2} \AA^{-1}$.

estimate because it appeared as an unresolved source. The NKSE mass model predicts that an object at the position of E1 with a redshift of 3.347 is just one of the five images of a multiply-imaged background object. A posteriori, a close inspection of the WFPC2 images reveals E1 as a compact unresolved component with a faint NW extension. The NKSE model predicted also the positions of the other four images: a close inspection of the WFPC2 data actually showed images very close to the expected locations(see Fig. 1), all of them with a morphology similar to E1 (Fig. 5) and apparently following the parity predicted by the lens model. Using E1, E2 and E5, the three images which are not contaminated by bright objects, we could verify, within the errors, that they actually have the same spectral energy distribution (SED), which is a necessary condition for gravitational images of the same source.
The discovery of the E-system is a very impressive success of the NKSE mass model for AC 114, and at the same time offers the possibility to improve the model. This is done in the next section.

\section{The lensing mass model revisited}

The NKSE lensing model has been revised in order to include the strong constraint imposed by the redshifts of two multiple-image systems: the 3 -image $\mathrm{S}(z=1.86)$, that had been already included in the NKSE model, and the newly discovered 5-image $\mathrm{E}(z=3.347)$.

We follow the NKSE assumptions in tuning up the mass model, and we refer to their work for more details. In summary, the mass distribution is well represented by a cluster-scale component, the central clump, and by 

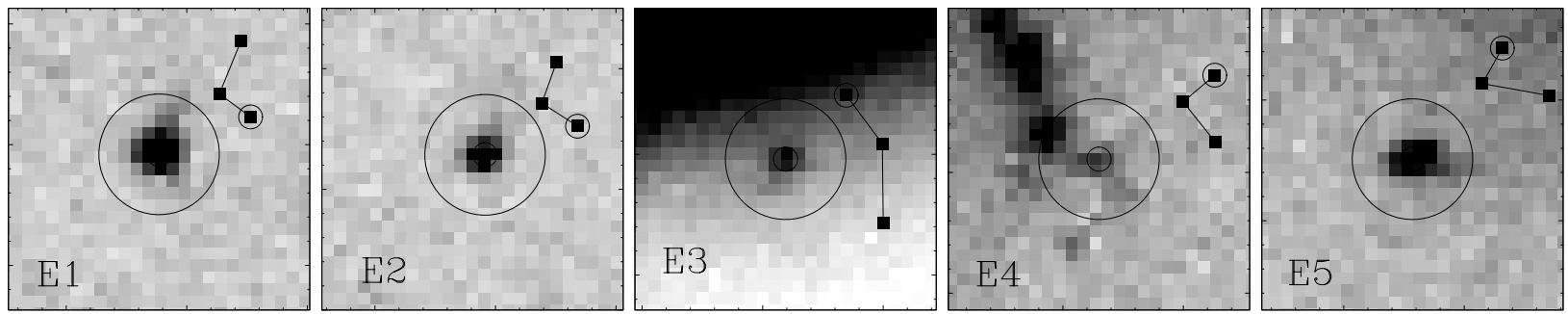

Fig. 5. Morphology of the 5 images of the multiple system E, labelled from E1 to E5. The size of each chart is $2.5^{\prime \prime} \times 2.5^{\prime \prime}$. We can identify on most charts a point source with 2 faint extensions. At the top right of each panel, a representation is given to show the expected parity of the extensions for all five images. Although the image resolution is not good enough to confirm the prediction, it seems that the elongations on each images are as expected from the lensing theory.

Table 3. Redshift determination for the 23 observed background galaxies. The horizontal line separates the core sample, as in Table 2. For objects with fair $\mathrm{S} / \mathrm{N}$ on the spectral continuum, the best fit spectral type is given, from $\mathrm{E}$ to $\mathrm{Im}$.

\begin{tabular}{|c|c|c|}
\hline Id. & $\begin{array}{c}z \\
\text { (spectra) }\end{array}$ & $\begin{array}{l}\text { Main Spectral } \\
\text { features }\end{array}$ \\
\hline A1 & 1.6912 & $\operatorname{MgII}(2798 \AA)$, FeII $(1608 \AA), \operatorname{AlII}(1670 \AA)$ \\
\hline $\mathrm{A} 2$ & 1.6912 & e. line $7518 \AA$ \\
\hline $\mathrm{B} 2$ & $1.50 / 2.08$ & e. line $4776 \AA$ \\
\hline B3 & $1.50 / 2.08$ & e. line $4776 \AA$ \\
\hline C3 & 2.854 & Ly $\alpha$, CIV $1549 \AA$ \\
\hline E1 & 3.3470 & $\operatorname{Ly} \alpha$ \\
\hline $\mathrm{S} 2$ & 1.8671 & blue cont., abs. lines (see text) \\
\hline V1 & $?$ & no clear feature \\
\hline $\mathrm{V} 2$ & 1.2143 & Im, $[\mathrm{OII}] 3727 \AA$ \\
\hline $\mathrm{V} 3$ & 0.706 & e. line $4776 \AA$ \\
\hline $\mathrm{V} 4$ & 2.050 & CIV $1549 \AA$ \\
\hline V5 & 1.0726 & S, e. line $7724.4 \AA$ \\
\hline V6 & 0.4095 & Im, [OII] $3727 \AA$ A, Balmer lines \\
\hline V7 & 0.5669 & Im, [OII] $3727 \AA[\mathrm{OIII}] 5007 \AA$, Balmer lines \\
\hline V8 & $0.58 ?$ & S, $4000 \AA$ break, faint abs. lines \\
\hline V9 & 0.4120 & Red S, [OII] $3727 \AA$ A, Balmer lines \\
\hline V10 & 0.8034 & Red S, [OII]3727 Å, Balmer lines \\
\hline V11 & 0.3805 & $\mathrm{Im},[\mathrm{OII}] 3727 \AA,[\mathrm{OIII}] 5007 \AA$ \\
\hline V12 & 0.7200 & 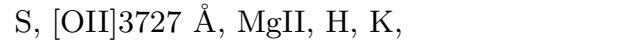 \\
\hline V13 & 0.7196 & $\mathrm{~S},[\mathrm{OII}] 3727 \AA$ \\
\hline V14 & 0.9003 & Im, [OII]3727 Å, Balmer lines, MgII \\
\hline V15 & 0.9023 & $\mathrm{E}, \mathrm{H}, \mathrm{K}$ \\
\hline V16 & $0.8994 ?$ & S, [OII] $3727 \AA$ \\
\hline V17 & 0.7117 & $\mathrm{~S}, \mathrm{H}, \mathrm{K}$, Balmer lines \\
\hline V18 & $0.8135 ?$ & $\mathrm{Im},[\mathrm{OII}] 3727 \AA$ \\
\hline
\end{tabular}

an additional bimodal cluster-scale component, with the clumps centered on the main galaxy concentrations. A galaxy-scale component is also introduced, with galaxy halos centered on each bright cluster galaxy, modelled by a pseudo-isothermal elliptical mass distribution, with parameters scaled to the galaxy luminosity (truncation and core radius, velocity dispersion). To improve the optimization of the LENSTOOL software (Kneib 1993), we used a new technique involving both a Monte-Carlo search as well as a parabolic minimization (Golse et al. 2001). Only the positions of the multiple images were fitted, and the free model parameters were the ellipticity, orientation, velocity dispersion, core radius of the cluster clump, and the ellipticity, orientation, velocity dispersion and truncation radius of the central $\mathrm{cD}$ galaxy. Table 4 summarizes the characteristics of the new revised model for the mass distribution. The best reduced $\chi^{2}$ found is $\sim 2$.

Using the best fitted mass distribution, we checked that the mean differences in amplification between the multiple images of the same object agreed with the ones observed. In particular for the E-system the measured values, $\Delta m_{\mathrm{E} 1-\mathrm{E} 2}=0.1 \mathrm{mags}$ and $\Delta m_{\mathrm{E} 2-\mathrm{E} 5}=0.7 \mathrm{mags}$, are in good agreement with model predictions.

We also used the revised NKSE model to check the redshift predictions for the other multiply-imaged objects in the cluster core. We found that the best redshift estimates for the multiple-image systems A, B, C and D are: $z_{\mathrm{A}}=1.7 \pm 0.05, z_{\mathrm{B}}=1.3 \pm 0.1, z_{\mathrm{C}}=2.3 \pm 0.1$ and $z_{\mathrm{D}}=1.4 \pm 0.1$. These values are larger than the ones previously predicted by NKSE (see Table 5), resulting in a better agreement between the $z_{\text {lens }}$ and the spectroscopic redshifts (see Sect. 6).

Table 5 lists the lensing redshifts for the multiplyimaged objects observed with VLT in the cluster core, and the predicted magnification for the 23 background galaxies observed with FORS1 (using the revised model of $\mathrm{AC} 114$ ) and the measured spectroscopic redshifts.

\section{Lensed galaxies in the cluster core}

The identification of spectral features and the main issues concerning the multiple images and the highly amplified galaxies are discussed in this section.

\subsection{The multiple image $A$ at $z=1.691$}

In this case, the redshift is mainly based on a single emission line, which is found in both the spectra of A1 and A2 at $7518 \AA$. This line is identified as MgII (at $2798 \AA$ ), in good agreement with the shape of the continuum. Besides, the spectrum of A1 also displays absorption lines, mainly FeII $(1608 \AA)$ and AlII (1670 $)$. A comparison between the spectra of A1 and the local starburst NGC 4214 
Table 4. Characteristics of the revised model for the mass distribution in AC 114. For each mass component, the following parametres are given: position, ellipticity, core radius $\left(r_{\mathrm{c}}\right)$, velocity dispersion and truncation radius $\left(r_{\mathrm{t}}\right)$. The galaxy-scale component is given for $L_{B}^{*}$.

\begin{tabular}{lccccccc}
\hline Mass Component & $\begin{array}{c}x \\
(\operatorname{arcsec})\end{array}$ & $\begin{array}{c}y / b \\
(\operatorname{arcsec})\end{array}$ & & $\begin{array}{c}\theta \\
(\mathrm{deg})\end{array}$ & $\begin{array}{c}r_{\mathrm{c}} \\
(\mathrm{kpc})\end{array}$ & $\begin{array}{c}\sigma \\
(\mathrm{km} / \mathrm{s})\end{array}$ & $\begin{array}{c}r_{\mathrm{t}} \\
(\mathrm{kpc})\end{array}$ \\
\hline Central Clump & $0.0 \pm 0.5$ & $0.0 \pm 0.5$ & $2.10 \pm 0.05$ & $14.5 \pm 0.5$ & $97 \pm 5$ & $1036 \pm 5$ & $2000 \pm 200$ \\
Clump 1 & $-105.0 \pm 10$ & $0.0 \pm 10$ & $1.250 \pm 0.1$ & $40 \pm 10$ & 100 (fixed) & $620 \pm 20$ & 1000 (fixed) \\
Clump 2 & $80.0 \pm 5$ & $-35.0 \pm 10$ & $1.350 \pm 0.1$ & $-30 \pm 10$ & 100 (fixed) & $450 \pm 20$ & 1000 (fixed) \\
$L_{B}^{*}$ Galaxy halo & - & - & - & - & 0.15 & $205 \pm 5$ & $25 \pm 5$ \\
\hline
\end{tabular}

(Leitherer et al. 1996) is given in Fig. 6. In addition, this redshift value is fully compatible with both the multipleimage configuration $(z=1.7 \pm 0.05$ derived from the revised lensing model) and the $z_{\text {phot }}$ (see Table 5 ). With $z=1.691$, we do not expect to find other strong emission lines, such as [OII] $3727 \AA$, CIV $1549 \AA$ or Ly $\alpha$, because they are outside the observed spectral range (Fig. 6).

\subsection{The multiple image $S$ at $z=1.867$}

The previous determination of the redshift of the system S1/S2 by Smail et al. (1995) is confirmed by the present results. We have obtained a spectrum for S2. Figure 7 displays the high $\mathrm{S} / \mathrm{N}$ spectrum as well as the complete line identification. A detailed study on this particular object will be presented in a further paper. This object is also important because it allows a (positive) test of the $z_{\text {phot }}$ techniques at $1 \leq z \leq 2$, a redshift domain which is particularly difficult to check because of the lack of strong spectral features.

\subsection{A new multiple image system (E) at $z=3.347$}

The initial redshift of the system was based on the spectrum taken for E1. The spectrum, shown in Fig. 8, shows a strong emission line which implies $z=3.347$ for the system when it is identified a Ly $\alpha$ line at $1216 \AA$. As already mentioned, the use of this redshift and the mass model for AC 114 predicted the additional 4 images of the system which are actually present with essentially the same morphology and SED. In fact, this system is one of the two new strong constraints of the new mass model presented in Sect. 5. Figure 9 shows the 2D spectra of E1 and A2+E4; E4 shows a very faint emission line at exactly the same wavelength as in E1, which is fully compatible with the lensing interpretation. The equivalent (rest frame) width of Ly $\alpha$ is $W_{\lambda}=61 \pm 7 \AA$. The error bar takes into account the uncertainties on the continuum level. The corrected absolute magnitude of the source galaxy, $M_{B}=-20.6$, together with the observed morphology, suggests a Seyfertlike galaxy.

\subsection{The multiple images $B$ and $C$}

We have obtained spectra for B2 and B3. A single emission line was found in both spectra, at $\lambda \sim 4776 \AA$. Photometric redshift could be obtained only for B2 and B1
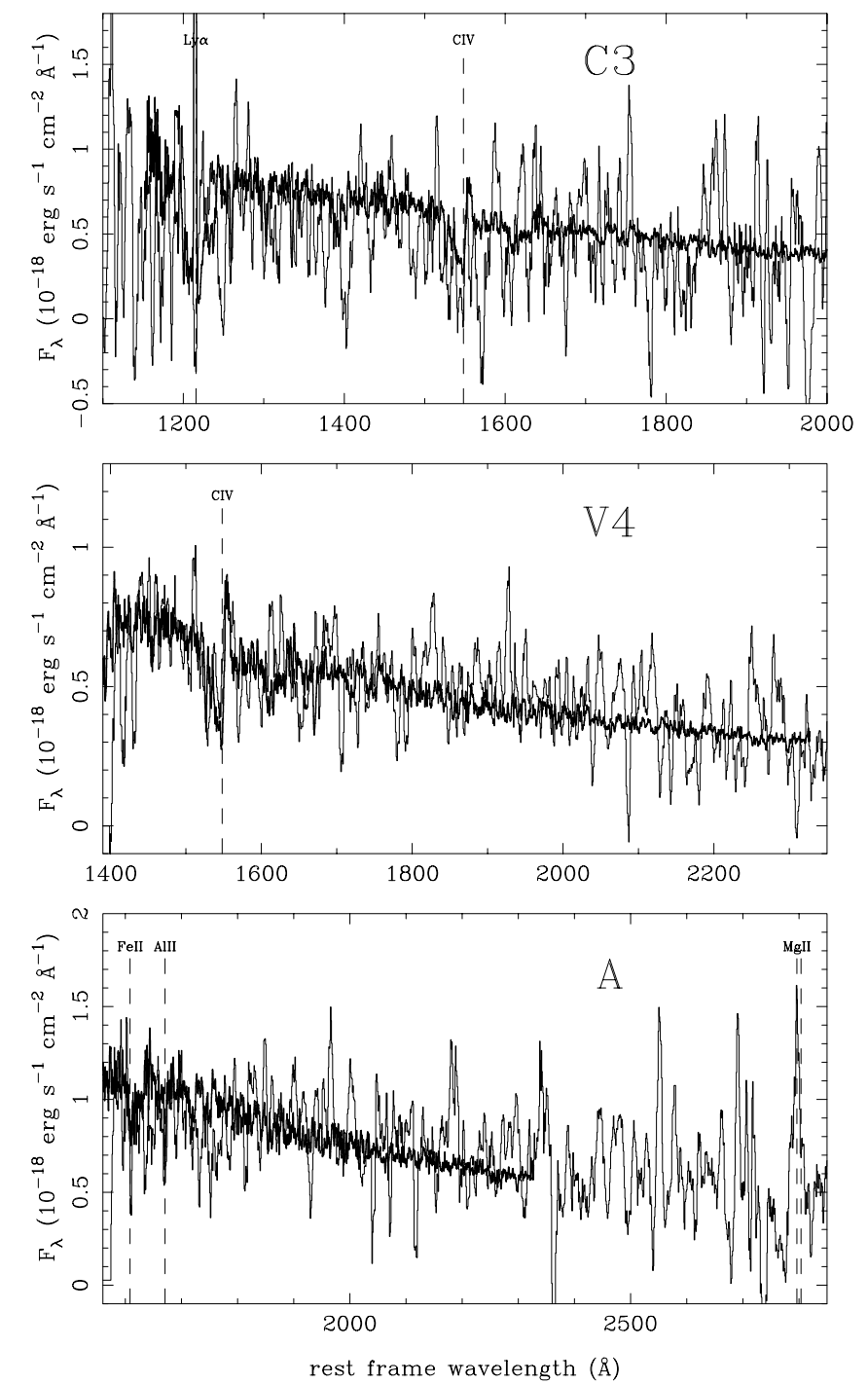

Fig. 6. From top to bottom, comparison between the spectra of C3 $(z=2.854), \mathrm{V} 4(z=2.050)$ and A1 $(z=1.691)$ (thin lines), and the local starburst galaxy NGC 4214 (Leitherer et al. 1996) (thick lines). The main spectral features are shown.

only, because B3 and B4 are contaminated by the neighbouring cluster galaxy on most of the ground-based images. The lensing and photometric redshifts for the image $\mathrm{B} 2$, are $z=1.3 \pm 0.1$ and $z=1.61_{-0.13}^{+0.19}$, respectively, at $1 \sigma$. The $z_{\text {phot }}$ for the image $\mathrm{B} 1$ is $z=1.57_{-0.25}^{+0.26}$, in 

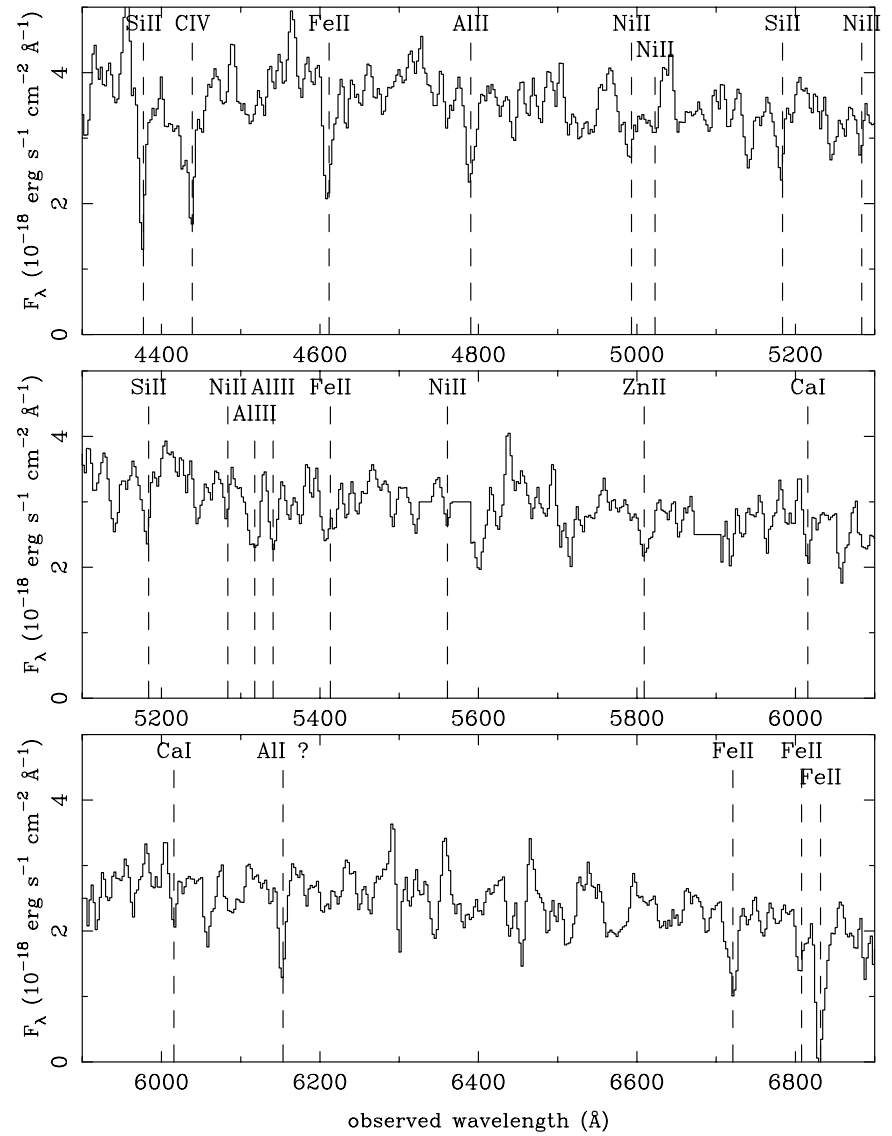

Fig. 7. Spectrum of object S2, at $z=1.867$, which confirms the identification of Smail et al. 1995. Note the blue excess of this galaxy.

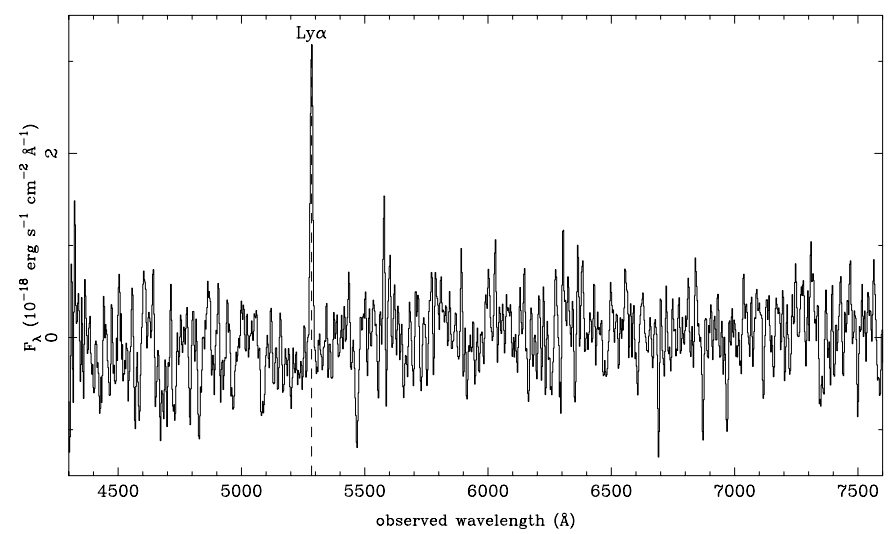

Fig. 8. Spectrum of object E1, at $z=3.347$, showing a strong emission line, identified as Ly $\alpha$. This redshift implies that E1 is part of a 5 -image system, which is actually verified. No other features are clearly identified on the continuum.

excellent agreement with B2. The only plausible identification for the emission line satisfying these two conditions is CIII] $1909 \AA$, implying a redshift of $z=1.502$. However, it does not fit exactly with the lensing prediction suggesting that either the line identification is not correct, or the lens model in this region is not accurate enough. The emission line is seen on the spectra of both B2 and B3+B4, as can be appreciated in the 2D spectra shown in Fig. 10,

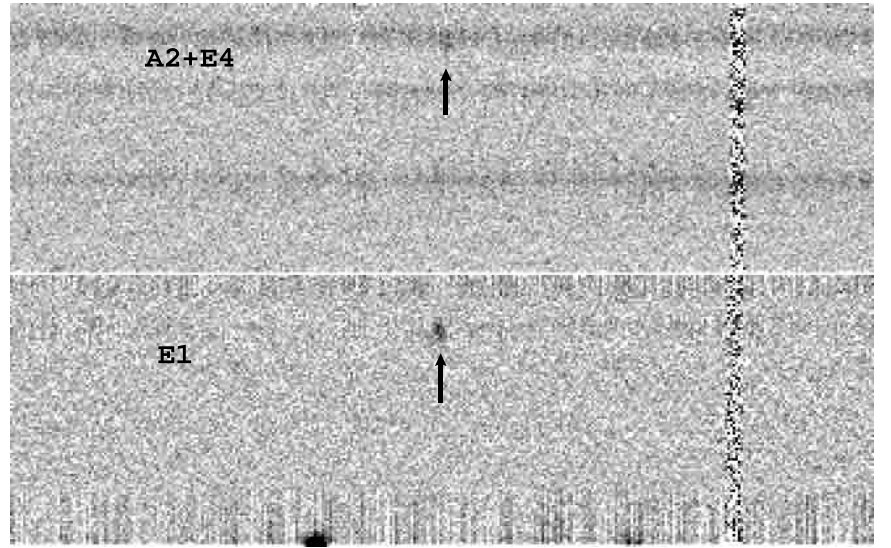

Fig. 9. The 2-dimensional spectra of two images of the galaxy E. The arrows indicate the position of Ly $\alpha$ line. The spectrum of the image E4 is blended with that of image A2 of $\operatorname{arc~A,~responsible~for~the~spectral~continuum~seen.~Note~the~}$ slight shift along the slit between continuum spectrum of A2 and the emission line of E4, which is compatible with their position and orientation on the HST image.

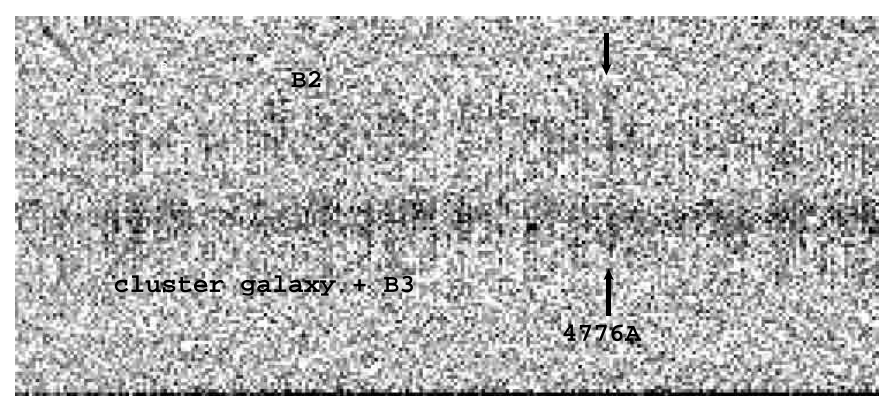

Fig. 10. The 2-dimensional spectra of two images of the arc B: B2 (top) and B3+B4 (bottom). The arrows point the position of a faint emission line, visible in both spectra of $\mathrm{B} 2$ and $\mathrm{B} 3+\mathrm{B} 4$ images. The B3+B4 spectrum is contaminated by light from a nearby cluster galaxy. The slit was placed on the alignment of the B2 image with B3 and B4 (see Fig. 1).

thus it is likely to be a real feature. When it is identified with CIV $1549 \AA$, which is a more likely case, then $z=2.08$, a value which is still compatible with the $z_{\text {phot }}$ at $2 \sigma$. Unfortunately, in this case Ly $\alpha$ is outside the spectral range observed. If the lens model in this region is responsible for the discrepancy, and the actual redshift of $B$ is higher than $z \sim 1.4$, then the redshift prediction for the system C, $z=2.3 \pm 0.1$, is also likely to be underestimated. Deeper spectroscopy in the $I-z$ bands may allow to detect the [OII] emision line, specially if the lensing prediction is correct.

Our spectrum of image C3 provides a fair estimate of the redshift of the multiple image $\mathrm{C}$, which shows a blue continuum with absorption features identified as Ly $\alpha$ and CIV $1549 \AA$. The redshift derived from these two lines is $z=2.854$, which is fully compatible with the $z_{\text {phot }}$ estimates. Figure 6 displays a comparison between the spectra of C3 and that of a local starburst. Such a spectroscopic redshift is however much larger than the present lens prediction, but in concordance with the results obtained for 
the multiple image B. Clearly better data on B and C are required before deriving any strong conclusion.

\subsection{The amplified galaxies $V 1, V 2, V 3, V 4$ and V5}

Four other, high redshift, singly-imaged galaxies were observed with FORS-1:

- V1 is the only source for which we could not identify a clear spectral feature, despite a long exposure time (2h $47 \mathrm{~m}$ ) using two different slit configurations. This object is faint and has a blue SED. According to its $z_{\text {phot }}$ determination, $z=1.13$, but no strong emission line was detected, in spite of the fact that [OII] $3727 \AA$ is expected at $\lambda \sim 8000 \AA$;

- V2 displays a single emission line, superimposed on a continuum spectrum, which leads to a redshift $z=$ 1.2143 when identified with [OII] $3727 \AA$. The $z_{\text {phot }}$ determination is in good agreement with this spectroscopic redshift;

- the only clear spectral feature in the spectra of V3 is an emission line at $\lambda=4776 \AA$. The $z_{\text {phot }}$ determination gives a $1 \sigma$ interval ranging between 0.81 and 0.96 , with a best fit at $z=0.84$. The most likely identification of this emission line is $\operatorname{MgII}(2798 \AA)$, leading to $z=0.706$, a value which is still compatible with the $z_{\text {phot }}$ determination at $3 \sigma$. Unfortunately, the [OII] $3727 \AA$ line, expected to lie close to the atmospheric [OI] $6300 \AA$ line, is hardly detected;

- the spectrum of V4 shows an emission line, with P-Cygni profile, and a blue continuum. According to its $z_{\text {phot }}$ determination, it is either a high- $z$ source, with $z=2.04$ ( $1 \sigma$ error bar ranging between $z=2.0$ and $z=2.07$ ), or a $z=0.29$ foreground galaxy (or even a cluster member within the errors). In the first case, the emission line is identified to CIV $1549 \AA$, leading to $z=2.050$. In the low $z$ case, the emission line is most likely identified with [OII] $3727 \AA$, and in this case $z=0.272$, leading to $M_{B}=-17.19$. The latter identification is difficult to reconcile with the morphology of this object, as it appears on the HST images. In addition, the comparison between this spectrum and that of a local starburst allows to identify other significant absorption features, which reinforces the high- $z$ identification (Fig. 6);

- V5 displays a single emission line, at $\lambda=7724.4 \AA$, and a relatively blue featureless continuum. According to its $z_{\text {phot }}$ determination, the emission line can be identified with [OII] $3727 \AA$, leading to $z=1.0726$.

\subsection{Galaxy luminosities and $W_{[\mathrm{OII}] \lambda 3727}$ 's in the spectroscopic sample}

The revised mass model has been used to derive the magnification of each of the images. The absolute magnitudes $M_{B}$ for these amplified galaxies are computed through a direct scaling of the observed SED, taking into account the spectroscopic $z$, and using the best-fit templates from

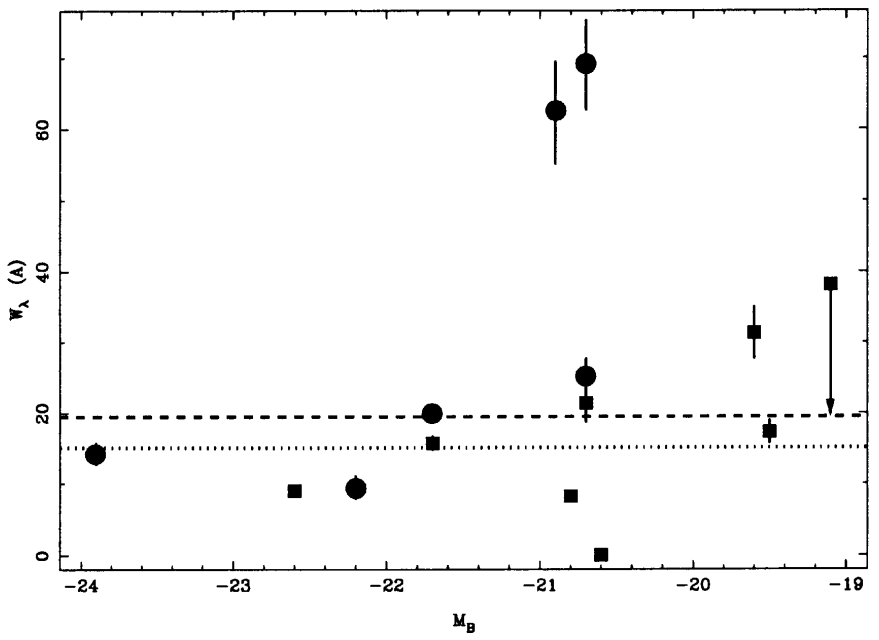

Fig. 11. Rest-frame equivalent width of $[\mathrm{OII}] \lambda 3727$ versus lens corrected $M_{B}$ in the spectroscopic sample of arclets at $0.4 \leq$ $z \leq 1.2$. Sources at redshifts lower and higher than $z=0.75$ are displayed as squares and filled dots respectively. Dotted and dashed lines show, respectively, the median values in the CFRS + LDSS sample at $0.5 \leq z \leq 0.75$, and the CFR sample at $0.75 \leq z \leq 1.0$ (Lilly et al. 1998).

the Bruzual \& Charlot code (Bruzual \& Charlot 1993) to derive the $k$-corrections. The $M_{B}$ values, corrected for amplification according to the revised NKSE model, are given in Table 5. The absolute magnitudes of these galaxies typically range from $M_{B} \sim-19$ to -22 , with a median value of -20.5 . In the cluster core, where the amplification is the highest, absolute magnitudes range between $M_{B} \sim-19$ and -21.5. In particular, $\mathrm{E}$ and $\mathrm{C}$, the two objects with confirmed redshifts in the interval $2.5 \lesssim z \lesssim 3.5$, are respectively 0.5 and 1.5 magnitudes fainter than the limiting magnitude of the Steidel et al. (1999) sample at similar redshifts. In the case of $\mathrm{S}$, the magnification corrected magnitude is close to the limiting value in conventional samples, but the magnification of $\sim 2$ magnitudes per image allows the obtention of spectra with higher signal-tonoise ratios.

We have computed the rest-frame equivalent width of [OII] $\lambda 3727\left(W_{\lambda}\right)$ for the 14 galaxies in the $0.4 \lesssim z \lesssim 1.2$ interval. There is no evidence for a correlation between the value of $W_{\lambda}$ and the redshift up to $z \sim 1$, but the 2 galaxies with the largest values of $W_{\lambda}(\geq 50 \AA)$ are also the ones at $z \geq 1$ (V2 and V5). There is no correlation between the flux in the emission-line and the redshift. The median value of $W_{\lambda}$ in our sample is $20 \AA$, a result which is fully compatible with the values found by Lilly et al. (1998) in the CFRS + LDSS sample at similar redshifts. Also, this value is similar to that obtained by Le Fèvre et al. (2000) for non-merger galaxies and upcoming mergers in the CFR sample. Figure 11 displays $W_{\lambda}$ versus $M_{B}$ for the 14 galaxies in the spectroscopic sample, compared to the median values obtained in the CFRS and the LDSS samples (Lilly et al. 1998). Even if the total number of sources is small, there is a clear trend in the sense that the faintest sub-L* galaxies $\left(M_{B} \geq-21\right)$ display the highest 
Table 5. Characteristics of the background galaxies studied in AC 114. Identifications are the same as in Table $2 . M_{B}$ have been corrected for magnification $\Delta m$ according to the revised NKSE model. The $z_{\text {lens }}$ values are from NKSE (1998). Error bars in $z_{\text {phot }}$ correspond to $1 \sigma$ using also a $B$-image. $M_{B}$ given in brackets correspond to objects for which the restframe $B$ band has been extrapolated from the available photometry, using the best fit model. It is highly model dependent in the case of V18.

\begin{tabular}{|c|c|c|c|c|c|c|c|}
\hline Id. & $\begin{array}{c}z \\
\text { (spectra) }\end{array}$ & $\begin{array}{c}z \\
\text { (phot.) }\end{array}$ & $\begin{array}{c}\Delta z \\
\text { (phot.) }\end{array}$ & $\begin{array}{c}z \\
\text { (lensing) }\end{array}$ & $\begin{array}{c}\Delta m \\
\text { mag. }\end{array}$ & $M_{B}$ & Comments \\
\hline $\begin{array}{l}\mathrm{A} 1 \\
\mathrm{~A} 2\end{array}$ & 1.6912 & 2.04 & $1.27-2.80$ & $1.67 \pm 0.15$ & 1.6(A1) & -20.5 & \\
\hline $\begin{array}{l}\text { B2 } \\
\text { B3 }\end{array}$ & \} $1.50 / 2.08$ & 1.61 & $1.48-1.80$ & $1.17 \pm 0.10$ & $1.8(\mathrm{~B} 2)$ & -19.5 & $M_{B}$ with $z=1.50$ \\
\hline C3 & 2.854 & 2.76 & $2.67-2.91$ & $2.1 \pm 0.3$ & 2.4 & -20.8 & \\
\hline E1 & 3.3470 & 3.45 & $3.29-3.60$ & - & 1.4 & -20.6 & \\
\hline $\mathrm{S} 2$ & 1.8671 & 2.06 & $1.97-2.25$ & - & 2.2 & -21.2 & \\
\hline $\mathrm{V} 1$ & $?$ & 1.13 & $1.05-1.22$ & & 1.4 & -19.9 & $M_{B}$ based on $z_{\text {phot }}$ \\
\hline $\mathrm{V} 2$ & 1.2143 & 0.945 & $0.41-1.08$ & & 0.8 & -20.9 & \\
\hline V3 & 0.706 & 0.84 & $0.81-0.96$ & & 0.5 & -19.1 & \\
\hline $\mathrm{V} 4$ & 2.050 & $0.29 / 2.04$ & $0.26-0.31 / 2.0-2.07$ & & 0.8 & -21.4 & \\
\hline $\mathrm{V} 5$ & 1.0726 & 1.05 & 0.91-1.47 & & 0.9 & -20.7 & \\
\hline $\mathrm{V} 6$ & 0.4095 & 0.46 & $0.38-0.50$ & & 0.1 & -19.5 & \\
\hline V7 & 0.5669 & 0.54 & $0.53-0.55$ & & 0.2 & -21.7 & \\
\hline V8 & $0.58 ?$ & 0.48 & $0.12-2.08$ & & 0.3 & -20.2 & \\
\hline $\mathrm{V} 9$ & 0.4120 & - & - & & 0.0 & -20.8 & \\
\hline V10 & 0.8034 & - & - & & 0.3 & -21.7 & \\
\hline V11 & 0.3805 & - & - & & 0.0 & -19.6 & \\
\hline V12 & 0.7200 & 0.51 & $0.373-0.59$ & & 0.2 & $(-22.6)$ & \\
\hline V13 & 0.7196 & 0.82 & $0.348-2.3$ & & 0.1 & $(-20.7)$ & \\
\hline V14 & 0.9003 & 1.26 & $0.54-3.29$ & & 0.1 & $(-20.7)$ & \\
\hline V15 & 0.9023 & - & - & & 0.1 & -22.7 & \\
\hline V16 & $0.8994 ?$ & - & - & & 0.1 & -22.2 & \\
\hline V17 & 0.7117 & - & - & & 0.0 & -20.6 & \\
\hline V18 & $0.8135 ?$ & - & - & & 0.0 & $(-23.9 ?)$ & \\
\hline
\end{tabular}

values of $W_{\lambda}$. According to Ellis et al. (1996), such a trend is also observed in the local samples. Besides, the evolution of the strong $[\mathrm{OII}] \lambda 3727$ emitters population $\left(W_{\lambda} \geq 20 \AA\right)$ is stronger than that observed for the whole population, i.e., the space density of such star-forming galaxies has strongly decreased since $z \sim 0.5$ (Ellis et al. 1996; Lilly et al. 1995). The results obtained on the present sample are in good agreement with the previous findings, and they extend the present samples in both redshift and towards the faintest end of the LF.

\subsection{Redshift distribution of lensed galaxies in the photometric sample}

One of the main goals of this program is to recover the intrinsic properties of a very faint subsample of high- $z$ lensed galaxies, undetectable otherwise, using deep images and $z_{\text {phot }}$, in order to investigate, in particular, their $z$ distribution and luminosity function. We have obtained through hyperz the $N\left(z_{\text {phot }}\right)$ distribution of arclets in the $1.2^{\prime} \times 1.2^{\prime}$ core of AC 114 , a region covered by all the images in Table 1 . Figure 12 displays the raw $N\left(z_{\text {phot }}\right)$ distribution for the 148 objects considered in this region. This sample includes objects detected in at least 3 filters. For $z_{\text {phot }}$ calculations, undetected objects in a given band have their flux in this band set to zero, with a flux error corresponding to the local limiting magnitude. Photometric errors are taken from SExtractor, with a threshold zeropoint error of 0.1 magnitudes. From this sample, we exclude all cluster-member candidates on the basis of their SEDs. Eight foreground and 81 background galaxies remain in the final sample, defined as objects excluded as cluster members to better than $90 \%$ confidence level, with $z \leq 0.2$ or $z \geq 0.4$. Forty-four galaxies are found at $1 \leq z \leq 7$ in a $1.2^{\prime} \times 1.2^{\prime}$ field centered on the $\mathrm{cD}$ galaxy. For comparison, the total number of such sources expected in this field, taking into account the depth of the survey, ranges from $\sim 30$ to 50 sources. The selection criteria allow that the background sample includes all the objects selected for spectroscopy in the cluster core.

For cluster lenses with well-constrained mass distributions, as in the present case, it is possible to recover precisely the $N\left(z_{\text {phot }}\right)$ and the absolute magnitude distributions of lensed galaxies by correcting the relative impact parameter on each redshift bin. We have used the NKSE corrected mass model to derive such distributions for the background sample of galaxies. Figure 13 displays the lens corrected absolute magnitude $M_{B}$ distribution versus redshift for the sources in the field of AC 114. Up to $z \sim 4$, the restframe $B$ band is well matched by the filters used here. 


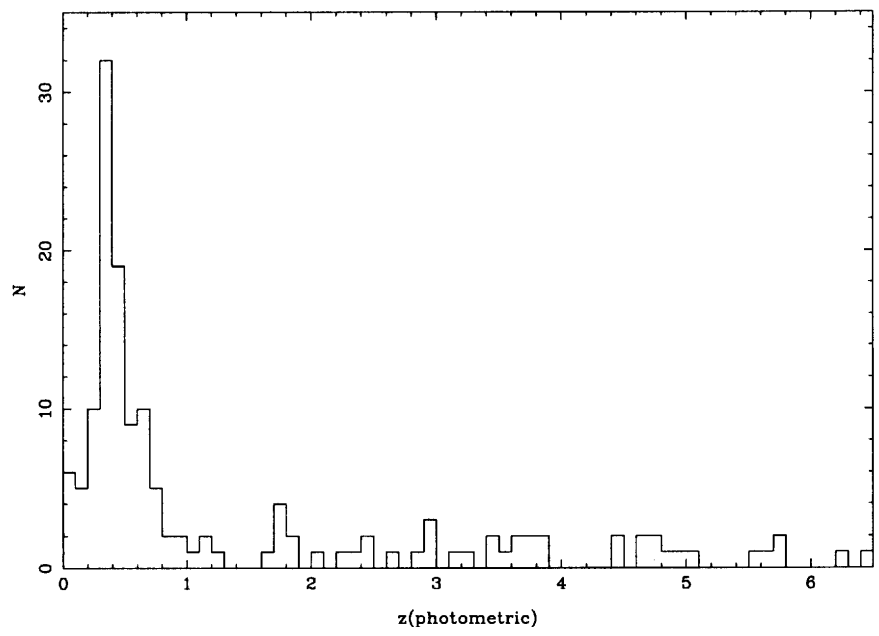

Fig. 12. Raw photometric redshift distribution of galaxies in the $1.2^{\prime} \times 1.2^{\prime}$ cluster core.

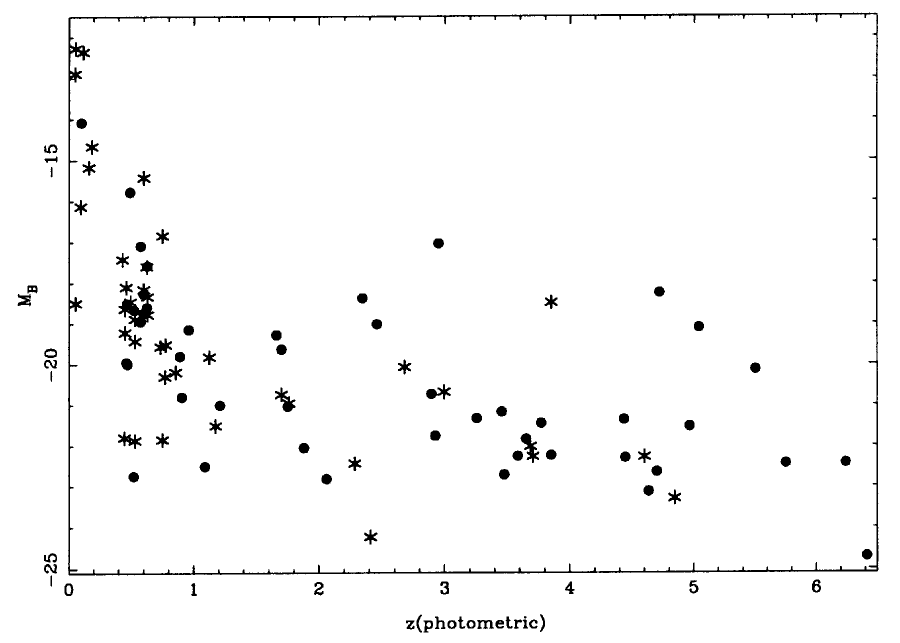

Fig. 13. Lens corrected absolute magnitude distribution versus redshift for sources in the $1.2^{\prime} \times 1.2^{\prime}$ cluster core. Black dots and stars correspond to objects with reduced $\chi^{2} \leq 1.5$ and $\chi^{2}>1.5$, respectively.

The median value of $M_{B}$ is -21.5 for the 44 galaxies at $1 \lesssim z \lesssim 7$, with typical values ranging between -19 and -23 . In the $1 \lesssim z \lesssim 3.5$ redshift interval ( 23 galaxies), the median $M_{B}$ is -21.0 . Thus, the spectroscopic sample is biased towards the most amplified sources in the central region, and thus intrinsically fainter than the photometric sample. The median intrinsic luminosity increases to $M_{B} \sim-22$ for the $z \geq 3.5$ sample. Because of the small number of objects in the sample, this exercise is of limited interest for a single cluster, when using standard (not ultra-deep) imaging, but it is useful to illustrate the case.

\section{Discussion}

This study has allowed us to improve the NKSE lensing model for the massive and well studied cluster AC 114, mainly through the discovery of a new multiple-image system, the E-system. This discovery was driven by the identification of a very high- $z$ galaxy in the core of
AC 114 following deep multi-band images, and specifically through the photometric redshift technique. This multiple-image system was missed by a search of lensed objects in the high-resolution HST images due to its pointlike appearance.

The way that led us to discover the E-system is a good demonstration of how high resolution images of cluster cores can be combined with deep multi-band images in order to select high- $z$ galaxy candidates and to further constrain cluster mass models. This could be achieved for a larger list of clusters, e.g. those in available archives. Following the example of AC 114, deeper and higher resolution images with diagnostic spectroscopy, will lead to new discoveries in the distant universe.

The photometry of a multiply-imaged background object together with the model predictions for the individual images should lead to a single value for the intrinsic luminosity of the source. The resulting typical uncertainty in the magnification factor is $\sim 0.3$ magnitudes for most objects, which gives an idea of the limitations arising from lens modelling when using gravitational telescopes.

The revision to the mass model of AC 114 is not a drastic one; only some parameters have been tuned to match the new constraints whereas the overall form is unchanged. However, if the redshifts of $\mathrm{B}$ and $\mathrm{C}$ systems are confirmed, then there will be a need for a major change to the model as these redshifts can only be matched if the mass profile is steeper than the one employed both in the original NKSE and the refined models. Once the redshifts of $\mathrm{B}$ and $\mathrm{C}$ are definitively known, an even more accurate model can be obtained and this cluster could be used with great confidence in other applications such as the search for distant supernovae (Sullivan et al. 2000), distant Submm sources (Smail et al. 1997) or Lyman- $\alpha$ emitters (Ellis et al., in preparation), or in the determination of the cosmological parameters $(\Omega, \lambda)$ using the method of Golse et al. (2001).

The source responsible for the multiple image $\mathrm{E}$ at $z=3.347$ is intrinsically faint, roughly the equivalent of the local $M_{B}^{*}+0.5$ magnitudes. The presence of a relatively strong emission line, together with the gravitational magnification, has allowed us to obtain a spectroscopic redshift. This galaxy is intrinsically $\sim 0.5$ magnitudes fainter than the limiting value of the Steidel et al. (1999) sample at similar redshifts. It has a bright core and at least two faint extensions, similar to the morphology of the compact cores in the field sample of Steidel et al. (1996a) at $2.5 \lesssim z \lesssim 3.5$. The main subclump displays a compact morphology, which is not resolved in width on the WFPC2 images, thus implying length scales of the order of $\sim 1 \mathrm{kpc}$. Its morphology and the equivalent (rest frame) width of Ly $\alpha$ are similar to those of $\mathrm{H} 5$, one of the multiple images at $z=4.05$ identified in the core of A2390 (Pelló et al. 1999), and also similar to the typical values observed by $\mathrm{Hu}$ et al. (1998) in their sample of emission line galaxies at $z \sim 3$ to 6 .

Six galaxies in our spectroscopic sample are found at $1 \lesssim z \lesssim 2.5$, a redshift domain which is relatively poorly explored because of the lack of strong spectral features. 
The gravitational amplification has allowed us to identify such objects, and the precise redshift determination, using visible and near-IR spectrographs in 8-10 m class telescopes, will allow a detailed study of the spectral energy distributions of these $z \geq 1$ galaxies. In particular, studies of the star formation rate history and of the permitted region in the age-metallicity-reddening parameter space, are now possible and will be presented in the future. The best example is the source $\mathrm{S}$, for which a detailed spectroscopic study is presently in progress.

The median value of $M_{B}$ is -21.5 for the photometric sample of galaxies at $1 \lesssim z \lesssim 7$, with typical values ranging between -19 and -23 . The median $M_{B}$ is -21.0 in this sample, in the $1 \lesssim z \lesssim 3.5$ redshift interval, and it increases to $M_{B} \sim-22$ for the $z \geq 3.5$ sample. The spectroscopic sample in the cluster core is biased towards the most amplified sources, which are intrinsically fainter than the photometric sample, and it exhibits a median $M_{B}$ of -20.5 in the $1 \lesssim z \lesssim 3.5$ redshift bin, with absolute magnitudes ranging between $M_{B} \sim-19$ and -21.5 . Lensed galaxies in this spectroscopic sample are found to be intrinsically fainter, between 0.5 and 1.5 magnitudes, than the limiting magnitudes in the present blank field surveys at $2 \lesssim z \lesssim 3.5$ (Steidel et al. 1999). This gain in sensitivity towards low luminosity high- $z$ objects, equivalent to an increase in collecting area by at least a factor of 2 to 3 (and up to a factor of $\sim 10$ in restricted regions of the source plane), is sufficiently large to allow considerable improvement on our knowledge of the faintest and/or highest redshift galaxies.

\section{Conclusions}

In summary, we have successfully identified 23 galaxies in the background of AC 114, 10 of them being high-redshift objects, with $0.7 \lesssim z \lesssim 3.5$, located in the cluster core. The main results of our spectroscopic survey, concerning the multiple-image systems and the mass model of $\mathrm{AC} 114$, are:

- We confirm the redshift $z=1.87$ of the gravitational pair S1/S2.

- The redshift of the multiple image system A has been measured: $z=1.69$, in excellent agreement with the lensing and photometric predictions.

- A new 5-image multiple system $\mathrm{E}$ at redshift $z=3.347$ is identified. We use this new strong constraint, together with the previously used multiple system S, to establish a revised mass model for AC 114.

- The redshifts of the multiply-imaged objects B and $\mathrm{C}$ are determined, however they need to be confirmed with new and better quality data. So far, their estimated values are slightly larger than those predicted originally by NKSE (1998), and are still difficult to reconcile with our revised model.

Based on the above mentioned results, we have shown the efficiency of a lensing/photometric approach to derive new samples of high- $z$ galaxies. In effect, all the highly magnified galaxies in the spectroscopic sample obtained in this work have $M_{B} \sim-21.5$ to -19 , thus opening a way for studies of the faint end slope of the luminosity function in early epochs of the universe. More specifically, the 2 objects $(\mathrm{C}, \mathrm{E})$ with spectroscopic $2.5 \lesssim z \lesssim 3.5$, are respectively 0.5 and 1.5 magnitudes fainter than the limiting magnitude of the Steidel et al. (1999) survey at similar redshifts. Also, we have shown that sub-L* galaxies in the $0.4 \lesssim z \lesssim 1.2$ interval tend to exhibit stronger restframe equivalent width of $[\mathrm{OII}] \lambda 3727$ than the brightest galaxies in the sample. This trend has already been observed in field surveys (Ellis et al. 1996; Lilly et al. 1995). The present sample extends the available data towards the faint of the $\operatorname{LF}\left(M_{B} \geq-21\right)$ at $z \geq 0.5$.

Probing the faint end of the luminosity function of high redshift galaxies will require observations and analyses, similar to the one presented here for AC 114, of about ten massive cluster-lenses.

Acknowledgements. We are grateful to M. Bolzonella, G. Bruzual, M. Dantel-Fort, G. Mathez and D. Schaerer for useful discussions on this particular program. We would like to thank W. J. Couch for providing his deep $B$ image of AC114, and C. Leitherer for allowing the use of the NGC 4214 spectrum. L.E.C. was partially supported by FONDECYT grant 1970735. Part of this work was supported by the French Centre National de la Recherche Scientifique, by the French Programme National de Cosmologie (PNC), and the TMR Lensnet ERBFMRXCT970172 (http://www.ast.cam.ac.uk/IoA/lensnet) and the ECOS SUD Program. Based on observations collected at the European Southern Observatory, Chile (ESO P64.O-0439), and with the NASA/ESA Hubble Space Telescope, which is operated by STScI for the Association of Universities for Research in Astronomy, Inc., under NASA contract NAS5-26555.

\section{References}

Abell, G. O., Corwin, H. G. Jr., \& Olowin, R. P. 1989, ApJS, 70,1

Altieri, B., Metcalfe, L., Kneib, J.-P., et al. 1999, A\&A, 343, L65

Arnouts, S., Cristiani, S., Moscardini, L., et al. 1999, MNRAS, 310,540

Barger, A. J., Aragón-Salamanca, A., Ellis, R. S., et al. 1996, MNRAS, 279, 1

Bertin, E., \& Arnouts, S. 1996, A\&AS, 117, 393

Bézecourt, J., Soucail, G., Ellis, R. S., \& Kneib, J.-P. 1999, A\&A, 351, 433

Bolzonella, M., Miralles, J. M., \& Pelló, R. 2000, A\&A, 363, 476

Broadhurst, T. J. 1995, preprint [astro-ph/9511110]

Bruzual, G., \& Charlot, S. 1993, ApJ, 405, 538

Ebbels, T. M. D., Ellis R. S., Kneib J. P., et al. 1998, MNRAS, 295,75

Ellis, R. S., Colless, M., Broadhurst, T. J., Heyl, J., \& Glazebrook, K. 1996, MNRAS, 280, 235

Fernández-Soto, A., Lanzetta, K. M., \& Yahil, A. 1999, ApJ, 513, 34

Fort, B., Mellier, Y., \& Dantel-Fort, M. 1997, A\&A, 321, 353 
Furusawa, H., Shimasaku, K., Doi, M., \& Okamura, S. 2000, ApJ, 534, 624

Giallongo, E., D'Odorico, S., Fontana, A., et al. 1998, AJ, 115, 2169

Golse, G., Kneib, J. P., \& Soucail, G. 2001, submitted to A\&A [astro-ph/0103500]

Gwyn, S. D. J., \& Hartwick, F. D. A. 1996, ApJ, 468, L77

Hu, E. M., Cowie L. L., \& McMahon, R. G. 1998, ApJ, 502, L99

Ivison, R. J., Smail, I., Barger, A. J., et al. 2000, MNRAS, 315, 209

Kneib, J.-P. 1993, Ph.D. Thesis, U. Paul-Sabatier

Kneib, J.-P., Mathez, M., Fort, B., et al. 1994, A\&A, 286, 701

Kneib, J.-P., Ellis, R. S., Smail, I., Couch, W. J., \& Sharples, R. 1996, ApJ, 471, 643

Lanzetta, K. M., Yahil, A., \& Fernández-Soto, A. 1996, Nature, 381,759

Le Fèvre, O., Abraham, R., Lilly, S. J., et al. 2000, MNRAS, 311,565

Leitherer, C., Vacca, W. D., Conti, P. S., et al. 1996, ApJ, 465, 717

Lilly, S. J., Tresse, L., Hammer, F., Crampton, D., \& Le Fèvre, O. 1995, ApJ, 455, 108

Lilly, S. J., Schade, D., Ellis, R. S., et al. 1998, ApJ, 500, 75

Mayen, C., \& Soucail, G. 2000, A\&A, 361, 415

Metcalfe, L., Altieri, B., McBreen, B., et al. 1999, in The Universe as seen by ISO, ed. P. Cox, \& M. F. Kessler, ESA Publication Division, ESTEC, Noordwijk, NL [astro-ph/9901147]
Mobasher, B., Rowan-Robinson, M., Georgakakis, A., \& Eaton, N. 1996, MNRAS, 282, L7

Natarajan, P., Kneib, J.-P., Smail, I., \& Ellis, R. S. 1998, ApJ, 499, 600 (NKSE)

Pelló, R., J.-P. Kneib, J.-F. Le Borgne, J., et al. 1999a, A\&A, 346,359

Pelló, R., Kneib, J.-P., Bolzanella, M., \& Miralles, J.-M. 1999b, Conference Proceedings of the Photometric Redshifts and High Redshift Galaxies, April 28-30, 1999, Pasadena [astro-ph/9907054]

Sawicki, M. J., Lin, H., \& Yee, H. K. C. 1997, AJ, 113, 1

Smail, I., Ellis, R. S., Fitchett, M. J., et al. 1991, MNRAS, 252, 19

Smail, I., Couch, W. J., Ellis, R. S., \& Sharples, R. M. 1995, ApJ, 440, 501

Smail, I., Ivison, R. J., \& Blain, A.W. 1997, ApJ, 490, L5

Steidel, C. C., Giavalisco, M., Pettini, M., Dickinson, M., \& Adelberger, K. 1996a, ApJ, 462, L17

Steidel, C. C., Giavalisco, M., Dickinson, M., \& Adelberger, K. 1996b, AJ, 112, 352

Steidel, C. C., Adelberger, K., Dickinson, M., et al. 1998, ApJ, 492,428

Steidel, C. C., Adelberg, K. L., Giavalisco, M., Dickinson, M., \& Pettini, M. 1999, ApJ, 519, 1

Sullivan, M., Ellis, R., Nugent, P., Smail, I., \& Madau, P. 2000, MNRAS, 319, 549 